\title{
Redesigned tetrads with altered hydrogen bonding patterns enable programming of quadruplex topologies $\dagger$
}

\author{
Armin Benz and Jörg S. Hartig* \\ Received (in Cambridge, UK) 27th March 2008, Accepted 18th July 2008 \\ First published as an Advance Article on the web 29th July 2008 \\ DOI: $10.1039 / \mathbf{b 8 0 5 2 2 7 a}$
}

Guanosine analogs assemble to unusual nucleobase tetrads, restricting possible conformations of four-stranded DNAs.

Guanine-rich nucleic acids are prone to fold into fourstranded structures called quadruplexes. Within such structures, four guanines engage in hydrogen bonds building a guanine tetrad. In quadruplex structures, such tetrads are stacked to form stable four-stranded secondary structures. ${ }^{1,2}$ While the core element, the guanine tetrad, is similar in all known guanine quadruplexes, the orientation of the participating strands differs significantly from one quadruplex to another and results in highly polymorphic folds. ${ }^{1,2}$ While RNA quadruplexes prefer conformations with all-parallel oriented strands due to the disfavored syn-conformation of the glycosidic bond, ${ }^{3}$ DNA quadruplexes display a variety of different topologies. Several factors affect DNA quadruplex conformations. The quadruplex topology is largely determined by the composition of the loops and the number of stacked G-tetrads. ${ }^{4}$ For example, sequences with short loops favor parallel orientation. ${ }^{5,6}$ For a given sequence, the conformation can strongly depend on the presence and type of cation. Probably the most characterized four-stranded sequence is the hexameric repeat found in vertebrate telomeres including those of humans. ${ }^{7}$ Four repeats are necessary to form an intramolecular quadruplex structure. Interestingly, several different topologies have been found. ${ }^{1,2}$ For example, a crystal structure in the presence of $\mathrm{K}^{+}$shows an all-parallel orientation, the so called propeller conformation. ${ }^{8}$ In addition, antiparallel orientation has been reported in $\mathrm{Na}^{+}$-containing solutions. ${ }^{9,10}$ More recently, in the presence of $\mathrm{K}^{+}$further structures were observed that contain mixed arrangements of both parallel and anti-parallel strand orientation. ${ }^{2,10,11} \mathrm{Circu}-$ lar dichroism is a powerful tool to distinguish different topologies. The all-parallel conformation displays a maximum of circular dichroism at $265 \mathrm{~nm}$, while folds containing antiparallel orientations are characterized by a maximum at $290 \mathrm{~nm}^{12}$

In the many different conformations adopted by quadruplexes, the terminal tetrads are built from different guanines. Upon exchanging guanosines with isostructural elements

University of Konstanz, Department of Chemistry and Konstanz Research School Chemical Biology (KoRS-CB), Universitätsstr. 10, 78457 Konstanz, Germany. E-mail: joerg.hartig@uni-konstanz.de; Fax: +49-7531-885140; Tel: +49-7531-884575

$\dagger$ Electronic supplementary information (ESI) available: Details of experimental procedures, analytical data, sequences, and further depictions of modified and mixed tetrads. See DOI: 10.1039/b805227a

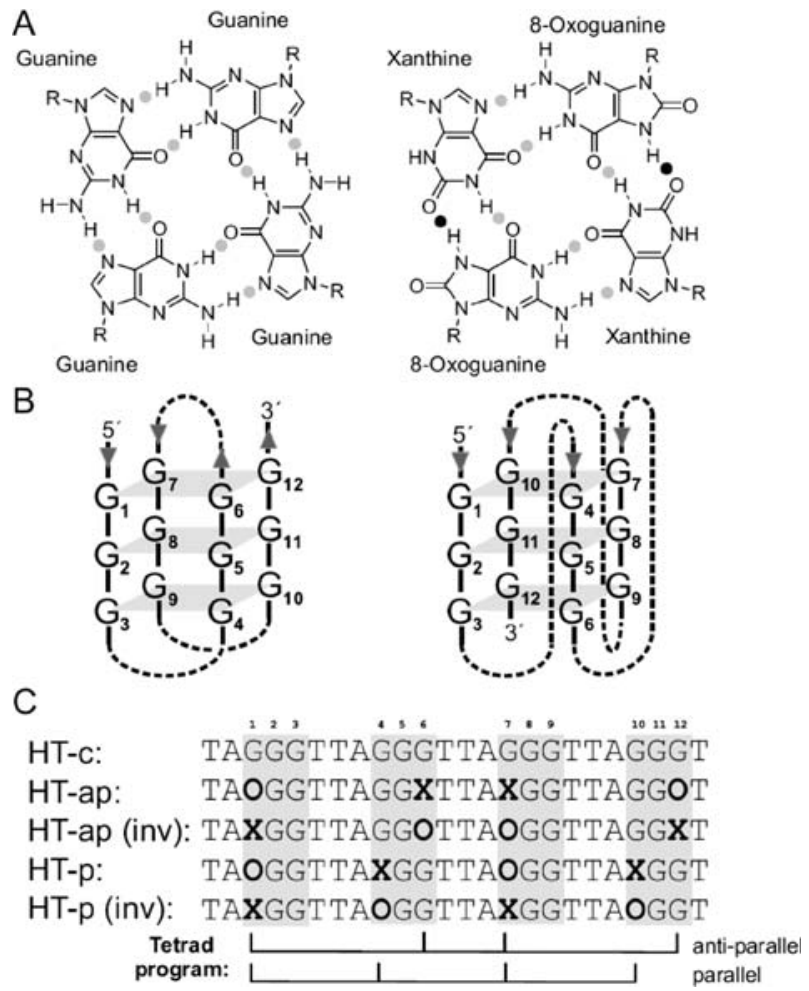

Fig. 1 Programmable quadruplex topologies. A: Comparison of tetrads composed of guanines (left) and 8-oxoguanines pairing with xanthines. The tetrads differ in the orientation of two hydrogen bonds (black dots). B: Two possible topologies of quadruplexes build by the human telomeric sequence. Left: anti-parallel topology, right: parallel topology. The loop connectivity is shown in dashed lines. C: Sequences characterized in this study. The positions of the 8-oxoguanosine (O) and xanthosine $(\mathbf{X})$ modifications should make it possible to selectively stabilize one topology compared to the other. 
natural counterpart. On the other hand, variation of the position of the guanosine analogs within the human telomeric repeat should make it possible to shift the preference of the quadruplex conformation towards a distinct topology. In parallel quadruplexes different guanosines participate in one tetrad compared to anti-parallel topologies. When designing a sequence that should fold into an anti-parallel topology, the alternative, parallel fold contains two tetrads each lacking two hydrogen bonds and vice versa (for a depiction of such destabilizing tetrads see ESI $\uparrow$ ). Hence, it should be possible to force the human telomeric sequence to adopt one fold or the other by programming hydrogen bonding patterns within tetrads composed of nucleobases other than guanosine.

The presented strategy should prove valuable if a specific conformation of a given sequence needs to be studied. For example, compounds binding to quadruplexes could be evaluated for their potential to differentiate between certain conformations. Recently described compounds differentiating between quadruplex structures have the potential to specifically target a quadruplex of interest leaving other naturally occurring G-rich sequences unaffected. ${ }^{13}$ In addition, the approach enables the functional characterization of individual quadruplex conformations.

In order to test our hypothesis, we have synthesized four sequences related to the human telomeric repeat that each contain two 8-oxoguanosine and two xanthosine modifications instead of guanosine, see Fig. 1C. Double NPE-protected, DMT-protected deoxyriboxanthosine phosphoramidite was synthesized according to published procedures. ${ }^{14}$ 8-Oxodeoxyguanosine phosphoramidite was commercially available and was incorporated together with the deoxyxanthosine building blocks into sequences HT-ap, HT-ap (inv), HT-p, and HT-p (inv) using standard solid phase oligonucleotide chemistry. The oligonucleotides were deprotected by treatment with $1 \mathrm{M}$ DBU followed by conventional ammonia cleavage and deprotection at $37{ }^{\circ} \mathrm{C} .{ }^{15}$ The oligonucleotides were purified by PAGE and subsequently analyzed by ESI-MS as well as analytical PAGE; for details of oligonucleotide synthesis see ESI. $\dagger$

Next, circular dichroism (CD) spectra were recorded in order to determine the predominant topology of the modified G-rich sequences. As a reference, an unmodified DNA strand composed of the vertebrate telomeric repeat (HT-c, see Fig. 1C) was analyzed. We chose potassium-containing buffer conditions that are known to favor the presence of an equilibrium of both parallel and anti-parallel topologies of the telomeric sequence. ${ }^{2,10,11}$ Indeed, the unmodified sequence displayed a maximum at $290 \mathrm{~nm}$ together with a pronounced shoulder at shorter wavelengths indicative of a mixture of antiparallel and parallel topologies, see Fig. 2A, black line. Interestingly, the modified sequence HT-p containing a tetrad program that should favor the parallel topology displayed a CD spectrum resembling a parallel fold whilst the second sequence HT-ap designed to adopt an anti-parallel conformation showed a clear shift to the anti-parallel fold. As a confirmation, the sequences HT-ap (inv) and HT-p (inv) were also characterized. The sequences differ by inversion of the respective 8-oxo-G and xanthosine positions, see Fig. 1C. The sequences should as well make it possible to program anti-

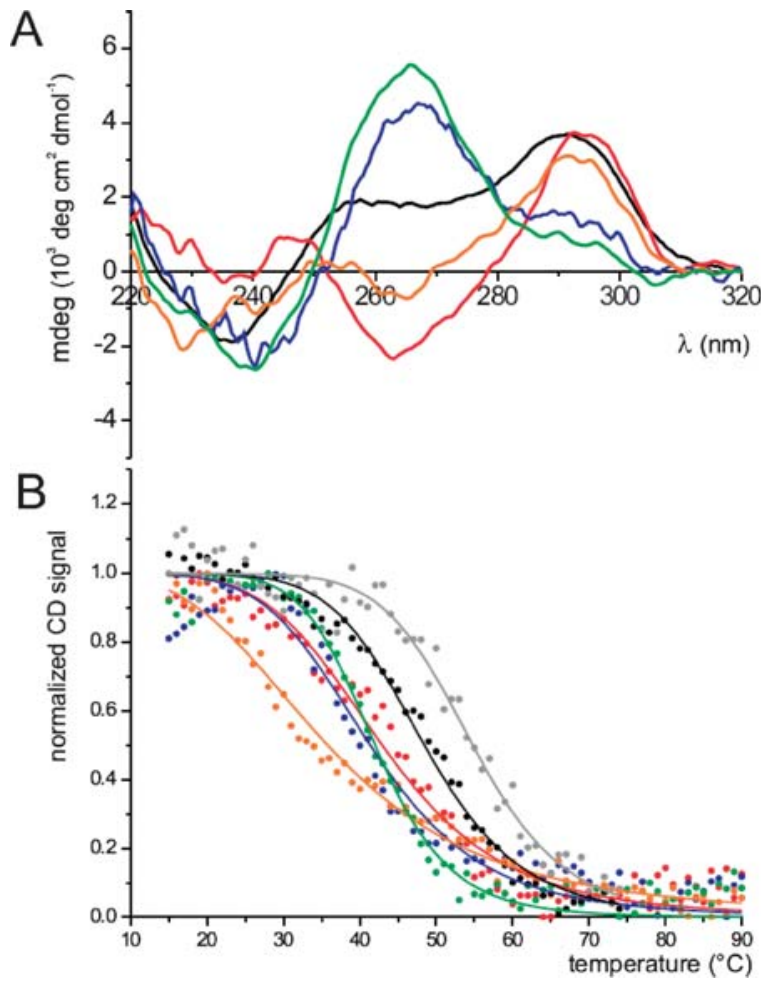

Fig. 2 Circular dichroism studies. A: CD spectra of the sequences HT-c: black; HT-ap: red; HT-ap (inv): orange; HT-p: blue; HT-p (inv): green; recorded in $10 \mathrm{mM}$ Tris, $\mathrm{pH} 7.5,25 \mathrm{mM} \mathrm{KCl}$ at $25{ }^{\circ} \mathrm{C}$. B: Thermal denaturation studies followed by $\mathrm{CD}$ spectroscopy. Sequence HT-c was observed at $265 \mathrm{~nm}$ (gray) and $290 \mathrm{~nm}$ (black), HT-p sequences at $265 \mathrm{~nm}$ and HT-ap sequences at $290 \mathrm{~nm}$. Buffer conditions were similar to $2 \mathrm{~A}$, data were normalized for better comparison.

parallel and parallel topologies. Indeed, the inverted sequences display similar CD spectra compared to HT-ap and HT-p, respectively. Hence, by redesigning the hydrogen bonding pattern of one tetrad by exchanging four guanines for two pairs of xanthines and 8-oxoguanines we succeeded in programming the overall topology of the quadruplex fold. In order to prove that indeed intramolecular quadruplexes are formed when a synthetic tetrad is present, we carried out gel shift experiments. All modified sequences display a band that corresponds to monomolecular quadruplexes although higherorder aggregates are visible as well (see ESI $\dagger$ ).

The CD spectra show a clear shift to the expected topologies. Nevertheless, especially the spectra of the anti-parallel sequences still display a slight shoulder around $290 \mathrm{~nm}$ corresponding to the disfavored topology. Hence it seems that there is still a minor fraction of the alternative fold present. In order to assess whether the redesigned tetrads interfere with the overall stability of the quadruplex, we carried out $\mathrm{CD}$ melting experiments. For comparison, the unmodified sequence HT-c was used. We observed the temperature-dependent decrease of the CD signal at both 265 and $290 \mathrm{~nm}$. This makes it possible to assess the stabilities of the anti-parallel as well as the parallel fold of the unmodified sequence, see Fig. 2B. Both folds showed significant stabilities represented by melting points of $49{ }^{\circ} \mathrm{C}$ for the anti-parallel conformation and of $55.5{ }^{\circ} \mathrm{C}$ for the parallel fold. In contrast, when the 
stability of the xanthine/8-oxoguanine-tetrad-containing quadruplexes was determined, both the parallel folds (observed at $265 \mathrm{~nm}$ ) and the anti-parallel folds (observed at $290 \mathrm{~nm}$ ) showed significantly lowered melting temperatures of $40{ }^{\circ} \mathrm{C}$ or below, see Fig. 2B. Although the incorporation of an 8-oxoguanine/xanthine-tetrad makes it possible to favor one quadruplex topology over the other, incorporation of the unnatural tetrad slightly destabilizes the quadruplex structure compared to a conventional tetrad made up from guanines.

In conclusion, the use of 8-oxoguanosine in combination with xanthosine makes it possible to program the overall topology of quadruplexes by redesigning constructive hydrogen bonding patterns. Notably, the strategy should even make it possible to program topologies very specifically. For example, the basket, chair, and dogear conformations of intramolecular quadruplexes differ in the guanosines participating in the external tetrads and hence should be individually programmable using this approach. The present study proves the viability of the concept whereas more elaborate structural studies will be necessary in order to differentiate between the mentioned conformations.

A variety of nucleobase analogs participating in quadruplex structures have been studied before. For example, Mergny and co-workers have characterized several guanosine derivatives in the context of tetrameric quadruplexes, finding mostly negative effects on stability and assembly kinetics of the four-stranded structures. ${ }^{16}$ In addition, 8-bromoguanosine-modified G-rich sequences showed altered quadruplex structures. ${ }^{17,18}$ Incorporation of adenosine analogs has been reported to result in changed conformations in tetrameric quadruplexes. ${ }^{19}$ Incorporation of ribonucleosides into DNA quadruplexes has been shown to restrict the possible conformations to parallel strand orientations. The likely reason for this observation is the preference of the anti-conformation of the ribonucleoside glycosidic bond. ${ }^{3}$ In contrast, substitutions in the 8-position favor the syn-conformation. ${ }^{20}$ Nevertheless, the anti-conformation of the glycosidic bond is still possible with guanosine analogs modified at C8. ${ }^{17,21}$ The parallel conformation of the natural telomeric sequence in its $\mathrm{K}^{+}$form contains only anticonformations of the glycosidic bond. ${ }^{8}$

The basket-type $\mathrm{Na}^{+}$form of the natural telomeric sequence displays alternating anti-syn-anti-syn conformations of guanines $1,6,7$, and 12 of the first tetrad referring to the numbering in Fig. 1., ${ }^{9,10}$ Since 8 -oxo-G favors the syn-conformation, one out of the two 8-oxo-Gs is in the disfavored anti-conformation in both HT-ap as well as HT-ap (inv). In the parallel form, both 8-oxo-Gs would adopt the disfavored glycosidic conformation. This would represent an explanation for the decreased stabilities of all modified quadruplexes compared to the natural sequence as shown in Fig. 2B. Nevertheless, the question of whether the structures of the modified, programmed sequences adopt the exact conformations of the natural sequences can only be addressed with more sophisticated structural studies. For a detailed review of the glycosidic conformations in vertebrate telomeric quadruplexes see ref. 2.

In addition, the presented findings are interesting with respect to the contribution of hydrogen bonds to the overall stability of quadruplex sequences. Since stacking interactions likely play a major role in stabilizing four-stranded sequences, ${ }^{22}$ it is remarkable that the difference of four out of in total 24 possible hydrogen bonds (eight per tetrad) is able to tip the folding equilibrium between anti-parallel and parallel quadruplexes to one side or the other (folding of the nonprogrammed conformation results in the loss of two hydrogen bonds in two tetrads together with potential steric hindrance in one tetrad, see ESI $\dagger$ ). Besides stabilizing factors such as nucleobase stacking and hydrogen bonding, electrostatic interactions play an important role, particularly in quadruplex structures. ${ }^{23}$ Hence, it will be interesting to characterize the behavior of the modified sequences with respect to different concentrations and types of cations. Anyway, only small differences in energetic stability of anti-parallel and parallel telomeric sequences have been found which could explain the viability of the presented approach. ${ }^{5,11}$

We thank Elmar Weinhold for sparking discussions, Astrid Joachimi and Vicki Lee for excellent technical assistance. JSH gratefully acknowledges the VolkswagenStiftung for funding a Lichtenberg-Professorship.

\section{Notes and references}

1 S. Burge, G. N. Parkinson, P. Hazel, A. K. Todd and S. Neidle, Nucleic Acids Res., 2006, 34, 5402.

2 D. J. Patel, A. T. Phan and V. Kuryavyi, Nucleic Acids Res., 2007, 35, 7429 .

3 C. F. Tang and R. H. Shafer, J. Am. Chem. Soc., 2006, 128, 5966.

4 P. A. Rachwal, T. Brown and K. R. Fox, Biochemistry, 2007, 46, 3036; A. Bugaut and S. Balasubramanian, Biochemistry, 2008, 47, 689.

5 P. Hazel, J. Huppert, S. Balasubramanian and S. Neidle, J. Am. Chem. Soc., 2004, 126, 16405.

6 P. A. Rachwal, T. Brown and K. R. Fox, FEBS Lett., 2007, 581, 1657.

7 R. K. Moyzis, J. M. Buckingham, L. S. Cram, M. Dani, L. L. Deaven, M. D. Jones, J. Meyne, R. L. Ratliff and J. R. Wu, Proc. Natl. Acad. Sci. U. S. A., 1988, 85, 6622.

8 G. N. Parkinson, M. P. Lee and S. Neidle, Nature, 2002, 417, 876.

9 Y. Wang and D. J. Patel, Structure, 1993, 1, 263.

10 A. T. Phan, V. Kuryavyi, K. N. Luu and D. J. Patel, Nucleic Acids Res., 2007, 35, 6517.

11 L. Ying, J. J. Green, H. Li, D. Klenerman and S. Balasubramanian, Proc. Natl. Acad. Sci. U. S. A., 2003, 100, 14629.

12 P. Balagurumoorthy and S. K. Brahmachari, J. Biol. Chem., 1994, 269, 21858; D. M. Gray, J. D. Wen, C. W. Gray, R. Repges, C. Repges, G. Raabe and J. Fleischhauer, Chirality, 2008, 20, 431; S. Paramasivan, I. Rujan and P. H. Bolton, Methods, 2007, 43, 324.

13 Z. A. Waller, P. S. Shirude, R. Rodriguez and S. Balasubramanian, Chem. Commun., 2008, 1467; A. Bugaut, K. Jantos, J. L. Wietor, R. Rodriguez, J. K. M. Sanders and S. Balasubramanian, Angew. Chem., Int. Ed., 2008, 47, 2677.

14 R. Eritja, D. M. Horowitz, P. A. Walker, J. P. Ziehler-Martin, M. S. Boosalis, M. F. Goodman, K. Itakura and B. E. Kaplan, Nucleic Acids Res., 1986, 14, 8135; A. Vanaerschot, M. Mag, P. Herdewijn and H. Vanderhaeghe, Nucleosides Nucleotides, 1989, 8, 159.

15 H. Sigmund and W. Pfleiderer, Helv. Chim. Acta, 2003, 86, 2299.

16 J. Gros, F. Rosu, S. Amrane, A. De Cian, V. Gabelica, L. Lacroix and J.-L. Mergny, Nucleic Acids Res., 2007, 35, 3064.

17 V. Esposito, A. Randazzo, G. Piccialli, L. Petraccone, C. Giancola and L. Mayol, Org. Biomol. Chem., 2004, 2, 313; Y. Xu, Y. Noguchi and H. Sugiyama, Bioorg. Med. Chem., 2006, 14, 5584.

18 L. Petraccone, I. Duro, A. Randazzo, A. Virno, L. Mayol and C. Giancola, Nucleosides, Nucleotides Nucleic Acids, 2007, 26, 669.

19 L. Petraccone, I. Duro, E. Erra, A. Randazzo, A. Virno and C. Giancola, Nucleosides, Nucleotides Nucleic Acids, 2007, 26, 675.

20 G. W. Hsu, M. Ober, T. Carell and L. S. Beese, Nature, 2004, 431, 217.

21 A. Virgilio, V. Esposito, A. Randazzo, L. Mayol and A. Galeone, Nucleic Acids Res., 2005, 33, 6188.

22 E. T. Kool, Acc. Chem. Res., 2002, 35, 936.

23 I. V. Smirnov and R. H. Shafer, Biopolymers, 2007, 85, 91. 